\title{
Phase transitions in cell migration
}

Caterina A. M. La Porta ${ }^{1,2, *}$ and Stefano Zapperi ${ }^{3,4, \dagger}$

${ }^{1}$ Center for Complexity and Biosystems,

Department of Environmental Science and Policy,

University of Milan, via Celoria 26, 20133 Milano, Italy

${ }^{2}$ CNR - Consiglio Nazionale delle Ricerche,

Istituto di Biofisica, via Celoria 26, 20133 Milano, Italy

${ }^{3}$ Center for Complexity and Biosystems,

Department of Physics, University of Milan,

Via Celoria 16, 20133 Milano, Italy

${ }^{4}$ CNR - Consiglio Nazionale delle Ricerche,

Istituto di Chimica della Materia Condensata e di Tecnologie per l'Energia,

Via R. Cozzi 53, 20125 Milano, Italy 
Phase transitions from a static to a moving phase are observed in a variety of physical systems and are thought to play a key role in cellular assemblies such as tissues or cancer. In this comment, we discuss how a suitable identification of control and order parameters can shed light on the nature of phase transitions in cell migration.

The epithelial-mesenchymal transition (EMT) is a program by which polarized epithelial (E) cells acquire mesenchymal $(\mathrm{M})$ characteristics. This is illustrated in Fig. 1a showing the key biological differences between these two cellular states: the presence/absence of cell polarity and the expression of adhesion molecules involved in mutual interactions and in the attachment to the extracellular matrix. These features account for the fact that $\mathrm{M}$ cells are able to migrate while E cells are stationary. The EMT can be reversible so that $\mathrm{M}$ cells can revert to the E state through the Mesenchymal to Epithelial Transition (MET). Recent evidence showed that the EMT can be a multiple-step process where cells express a mix of $\mathrm{E}$ and $\mathrm{M}$ features, giving rise to hybrid E/M states [1]. The EMT plays an important role in physiological processes such as development and would healing as well as in pathological conditions such as cancer dissemination. The capability of a cell to change its phenotype, when undergoing EMT or MET, is usually defined as cell plasticity and is nowadays one of the hallmarks of cell biology.

The transition of soft materials, such as colloidal suspensions, gels or foams, into a flowing state is often hindered by kinematic constrains responsible for jamming [2]. In the jamming state internal motion is prevented and the material behaves as an elastic solid, while in the unjammed state it behaves like a fluid. Experimental observations [3] suggest that the jamming-unjamming transition (JUT) and its reverse unjamming-jamming transition (UJT) could be relevant to describe the collective dynamics in cell monolayers and tissues or in cancer (for a review see [4]).

Several recent studies explored in depth each the two classes of transitions (EMT/MET or JUT/UJT) but important questions still remain unanswered. For instance, it is not clear if the two transitions are at all related, and in that case how. If the the two transitions are unrelated, we should understand whether they can work together or if they are completely independent or possibly mutually exclusive. We will illustrate here that a satisfactory answer to these questions can be obtained if we frame these biological observations in the broad context of the physics of non-equilibrium phase transitions and critical phenomena. To this 
end, we need to identify the phase space in which the transition takes place, the control parameters driving the transition and the order parameters describing the different phase.

While EMT/MET and JUT/UJT share a similar final outcome in terms of the mobilisation/immobilization of the cells, they are fundamentally different in terms of the topology of the phase space in which they occur. In analogy to their counterparts in soft materials, JUT/UJT occur in a physical space, such as a tissue or a cellular assembly in vitro. The relevant degrees of freedoms are the positions and velocties of the cells themselves. Possible control parameters are the cell density, the adhesion strength, the intensity of the cell traction forces and so on. While these control parameters are generally considered to determine the final state of cellular assemblies, the order parameter that would best describe the state is still debated. In our opinion, the order parameter should reflect the dynamic nature of the JUT/UJT and thus be constructed from the velocities or the trajectories of the cells. For instance, the velocity field [5] or the effective diffusion constant $D$ [6] can be used to distinguish a jammed from an unjammed phase. We may then need additional order parameters to characterize distinct active phases. Recent experiments on cancer cells, both in vitro and in vivo, revealed that JUT phase transitions between jammed solid, active fluid and active nematic [7] phases could be tuned controlling density and adhesion (see Fig. 1b) and described by measuring fluctuations in the velocity and vorticity $(\Omega=\nabla \times \mathbf{v}\rangle)$ fields [8].

On the other hand, EMT/MET occur within the biochemical phase space of individual cells. The relevant degrees of freedom are proteins whose abundances is described by a complex regulatory network [9]. Simulations of network models for the EMT/MET allows to map all the possible $\mathrm{E}$ and $\mathrm{M}$ states into a rugged pseudo-energy landscape (Fig. 1a). EMT/MET appear then as first order phase transitions driven by a multitude of control parameters that can be biochemical, as when the activity of key genes is perturbed, or physical, as when cells are deprived from oxygen. A possible order parameter for the EMT/MET is the expression of E-cadherin, a key adhesion molecule, or a more sophisticated measure of the position within the E/M landscape (Fig. 1a).

In conclusions, EMT/MET and JUT/UJT represnt two fundamentally different types of transitions, occurring within two separate phase spaces. Cells undergoing EMT can induce a JUT in the assembly through the reduction of cell-cell adhesion, a control parameter driving the JUT. The reverse is, however, not true since cells becoming jammed do not necessarily 
increase their mutual adhesion through a MET, an observation originally described as the "adhesion paradox" [3]. In light the discussion above, it turns out that this is not a paradox after all.

\section{Acknowledgments}

We thank for hospitality Ludwig-Maximilian University Munich and the Internationales Begegnungszentrum München where this work was completed. SZ also acknowledges support from the Alexander von Humboldt foundation through the Humboldt Research Award and thanks Friedrich-Alexander-Universität Erlangen-Nürnberg for hospitality.

\section{Competing interests}

Authors declare no conflicts of interest.

* Electronic address: caterina.laporta@unimi.it

$\dagger$ Electronic address: stefano.zapperi@unimi.it

[1] Grosse-Wilde, A., Fouquier d'Hérouël, A., McIntosh, E., Ertaylan, G., Skupin, A., Kuestner, R. E., del Sol, A., Walters, K.-A., and Huang, S. Stemness of the hybrid epithelial/mesenchymal state in breast cancer and its association with poor survival. PLoS One 10(5), e0126522 (2015).

[2] Liu, A. J. and Nagel, S. R. Jamming is not just cool any more. Nature 396(6706), 21-22 (1998).

[3] Park, J.-A., Kim, J. H., Bi, D., Mitchel, J. A., Qazvini, N. T., Tantisira, K., Park, C. Y., McGill, M., Kim, S.-H., Gweon, B., et al. Unjamming and cell shape in the asthmatic airway epithelium. Nature materials 14(10), 1040-1048 (2015).

[4] La Porta, C. A. and Zapperi, S. Cell Migrations: Causes and Functions, volume 1146. Springer, (2019).

[5] Garcia, S., Hannezo, E., Elgeti, J., Joanny, J.-F., Silberzan, P., and Gov, N. S. Physics of active jamming during collective cellular motion in a monolayer. Proceedings of the National Academy of Sciences 112(50), 15314-15319 (2015). 
[6] Bi, D., Yang, X., Marchetti, M. C., and Manning, M. L. Motility-driven glass and jamming transitions in biological tissues. Physical Review X 6(2), 021011 (2016).

[7] Saw, T. B., Doostmohammadi, A., Nier, V., Kocgozlu, L., Thampi, S., Toyama, Y., Marcq, P., Lim, C. T., Yeomans, J. M., and Ladoux, B. Topological defects in epithelia govern cell death and extrusion. Nature 544(7649), 212-216 (2017).

[8] Ilina, O., Gritsenko, P. G., Syga, S., Lippoldt, J., Porta, C. A. M. L., Chepizhko, O., Grosser, S., Vullings, M., Bakker, G.-J., Starruss, J., Bult, P., Zapperi, S., Käs, J. A., Deutsch, A., and Friedl, P. Cell-cell adhesion and 3d matrix confinement determine jamming transitions in breast cancer invasion. Nature Cell Biology in press (2020).

[9] Font-Clos, F., Zapperi, S., and La Porta, C. A. M. Topography of epithelial-mesenchymal plasticity. Proc Natl Acad Sci U S A 115(23), 5902-5907 (2018).

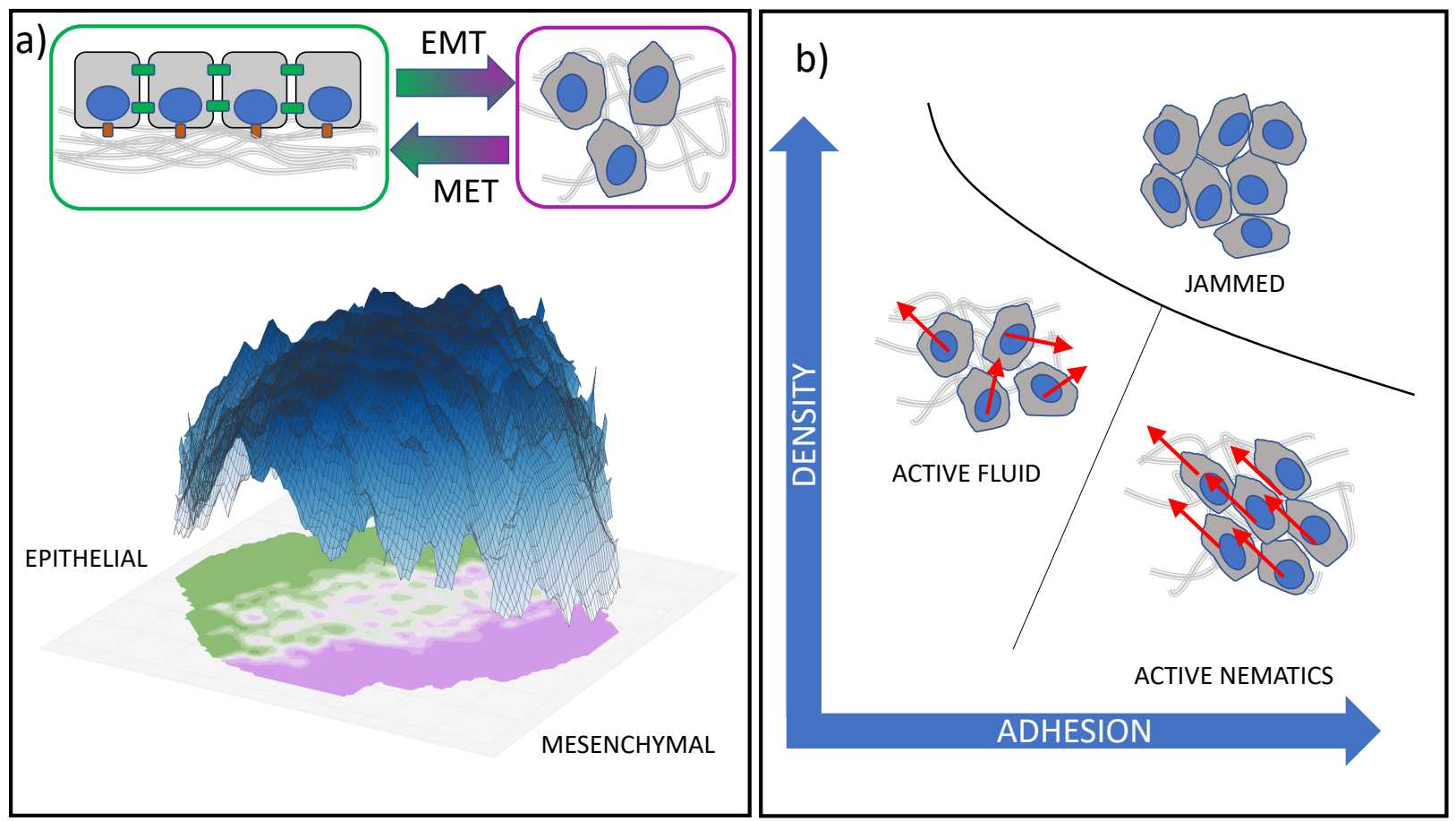

FIG. 1: Phase transitions. a) EMT/MET are transitions between epithelial and mesenchymal states. The phase space depicted here is a $2 \mathrm{D}$ projection of a multidimensional phase space representing the state of a set of genes in a complex regulatory network. The landscape shows the presence of a large number of metastable hybrid states, typical of first order phase transitions [9]. b) The phase diagram of the JUT/UJT depends on a number of control parameters, including cell density and adhesion [8]. EMT leads to the loss of adhesion and therefore may lead to JUT. 